\title{
Quantitative scoring of an interferon- $\gamma$ assay for differentiating active from latent tuberculosis
}

\author{
J-P. Janssens*, P. Roux-Lombard ${ }^{\#}$, T. Perneger", M. Metzger*, \\ R. Vivien ${ }^{\#}$ and T. Rochat*
}

ABSTRACT: The aim of this study was to assess the contribution of an interferon- $\gamma$ release assay (T-SPOT.TB) to the differentiation of active tuberculosis (TB) from latent TB infection by quantifying spot-forming units (sfu).

The investigation was a prospective study of contacts exposed to a case of contagious TB and cases of HIV-negative culture-proven TB referred over a 16-month period. Tuberculin skin tests (TSTs) and T-SPOT.TB were performed in 310 contacts 8-12 weeks after exposure. In subjects with culture-proven TB, T-SPOT.TB was performed within 2 weeks of initiation of treatment. The analysis included all contacts with a positive T-SPOT.TB result and all subjects with TB.

TB contacts $(n=127)$ and cases $(n=58)$ were included. Mean \pm SD T-SPOT.TB results were $107 \pm 56$ (range 1-207) sfu for TB, $54 \pm 60$ (7-239) sfu for contacts with positive T-SPOT.TB results and a TST induration diameter of $>5 \mathrm{~mm}$, and $19 \pm 27(7-143)$ sfu for contacts with positive T-SPOT.TB results and a TST induration diameter of $\leqslant 5 \mathbf{m m}$. By receiver operating characteristic curve analysis, a threshold value of 49.5 sfu showed a sensitivity of $83 \%$ and specificity of $74 \%$ for distinguishing latent TB infection from TB.

Although T-SPOT.TB results were significantly related to disease activity, the test cannot be recommended for the diagnosis of tuberculosis.

KEYWORDS: Interferon- $\gamma$ release assays, latent tuberculosis infection, tuberculosis

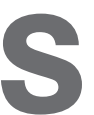
ince the early 1930s until the early 2000s, the only tool available for the detection of latent tuberculosis (TB) infection (LTBI) was the tuberculin skin test (TST). Although reactivity to the TST is associated with a welldocumented lifetime risk of developing active TB, one of the major limitations of the TST is its poor specificity (Sp); false-positive reactions, especially in bacille Calmette-Guérin (BCG)vaccinated populations, lead to potentially unnecessary preventive treatment for alleged LTBI. Since the early 2000s, the development of in vitro blood tests measuring production of interferon (IFN) $-\gamma$ by T-cells exposed to antigens highly specific to Mycobacterium tuberculosis (6-kDa early secretory antigenic target (ESAT-6) and culture filtrate protein (CFP) 10) has been an important advance in the detection of LTBI. These tests (T-SPOT.TB; Oxford Immunotec, Oxford, UK and QuantiFERON®-TB Gold; Cellestis, Carnegie, Australia), collectively referred to as IFN- $\gamma$ release assays (IGRAs), are affected by neither prior BCG vaccination nor most environmental mycobacteria (with the exception of Mycobacterium marinum,
Mycobacterion szulgai and Mycobacterion kansasii), thus offering increased Sp compared to the TST [1]. Their sensitivity (Se), extrapolated from studies in patients with active $\mathrm{TB}$, is probably at least as good as that of the TST in the detection of LTBI $[2,3]$. Studies comparing the TST and IGRA in contact-screening settings have consistently shown a higher correlation with exposure scores for the IGRA than for the TST [4-6]. Recent guidelines have integrated the use of IGRAs in screening strategies for subjects exposed to TB. Centers for Disease Control and Prevention (CDC) guidelines [7] state that the Quanti-

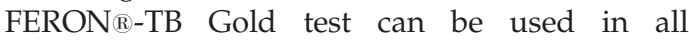
circumstances in which the TST is used and can be used in place of the TST (T-SPOT.TB did not have USA Food and Drug Administration approval when these guidelines were elaborated). The UK National Institute for Health and Clinical Excellence (NICE) 2006 guidelines [8] and the 2007 Swiss national guidelines [9] both recommend, for immunocompetent adults, a two-step procedure, i.e. confirmation of TST results considered positive using an IGRA.
AFFILIATIONS

Divisions of *Pulmonary Diseases,

${ }^{\#}$ Immunology and Allergy, and

-Quality of Care, Geneva University

Hospital, Geneva, Switzerland.

CORRESPONDENCE

J-P. Janssens

Centre antituberculeux

Hôpital Cantonal Universitaire

1211 Geneva 14

Switzerland

Fax: 41223729929

E-mail: Jean-Paul.Janssens@

houge.ch

Received:

March 112007

Accepted after revision:

May 142007

SUPPORT STATEMENT

This study was supported by the Geneva Pulmonary League (Geneva,

Switzerland), a not-for-profit

association which supports clinical

work and research in the field of

pulmonary disorders, particularly TB.

The Geneva Pulmonary League

approved the study protocol but had

no practical involvement in study

design, planning or data analysis.

STATEMENT OF INTEREST

None declared.

European Respiratory Journal

Print ISSN 0903-1936

Online ISSN 1399-3003 
One concern in contact screening is the detection of possible cases of TB among subjects with LTBI (defined either by a TST induration diameter of $>5 \mathrm{~mm}$ or a positive IGRA result). Indeed, the prevalence of TB in close contacts of TB cases is $\sim 1-2 \%$ ( $>100$-fold higher than in the general population) [10]. Several observations suggest a possible contribution of IGRAs in detecting TB in this setting. Indeed, a relationship between activity of $M$. tuberculosis disease and results of IGRAs has been documented by several studies. AIKEN et al. [11] showed a significant decrease in enzyme-linked immunospot (ELISPOT) count for both ESAT- 6 and CFP-10 antigens in patients with TB tested on diagnosis and 12 months later. Other studies have shown a marked reduction in the number of spot-forming units (sfu) produced by lymphocytes incubated overnight with the ESAT-6 peptide in patients with $\mathrm{TB}$ responding to treatment [12-14].

It was therefore hypothesised that, using T-SPOT.TB results (i.e. sfu number) as a continuous measure, it would be possible to establish a threshold sfu number permitting the distinction of TB from LTBI, in immunocompetent adults with a positive IGRA result. Thus, a prospective study was designed in order to compare T-SPOT.TB results in contacts screened for exposure to $\mathrm{TB}$ and patients admitted to Geneva University Hospital (Geneva, Switzerland) for TB during the same period.

\section{PATIENTS AND METHODS}

The outpatient clinic of the Division of Pulmonary Diseases of Geneva University Hospital follows most cases of TB and supervises all contact-tracing procedures for TB in the Geneva area $\left(245 \mathrm{~km}^{2} ; 440,000\right.$ inhabitants; annual TB incidence 20 cases per 100,000 population). Between October 1, 2004 and February 1, 2006, all patients with TB and all subjects screened for contact with a case of active TB were prospectively invited to participate in the present study. For subjects with TB, inclusion criteria were positive culture for M. tuberculosis and negative test result for HIV. For subjects screened for contact with a case of TB, systematic testing for HIV was not performed, but patients with a history of HIV infection were excluded from analysis. Patients with prior active TB were also excluded. The study protocol was accepted by the ethics committee on clinical research of Geneva University Hospital. All subjects included provided written informed consent.

Contacts were interviewed by a research nurse 8-12 weeks after their last exposure to the index case. The nurse completed a questionnaire, including details of: age; social status; country of birth; history of prior TST, HIV and TB infection and BCG vaccination; presence of BCG scars; and degree of infectiousness of the index case. A TST was performed, according to the Mantoux technique, using $2 \mathrm{U}$ of purified protein derivative of tuberculin (PPD) RT 23 (Statens Serum Institute, Copenhagen, Denmark; bioequivalent to $5 \mathrm{U}$ of the US PPD standard) and read after $72 \mathrm{~h}$ by two experienced nurses (induration diameters of readings were averaged). Blood sampling was performed for determination of $M$. tuberculosisspecific IFN- $\gamma$ secreting T-cells (T-SPOT.TB; Oxford Immunotec) at the same time as the TSTs. All subjects with a TST induration diameter of $>5 \mathrm{~mm}$ and/or a positive T-SPOT.TB test result were referred for clinical examination and chest radiography. Treatment for LTBI was recommended in all asymptomatic subjects with a TST induration diameter of $>5 \mathrm{~mm}$.

Subjects with TB had a blood sample taken for T-SPOT.TB analysis within 2 weeks of initiation of treatment. TST was not performed in subjects with TB.

Peripheral venous blood samples $(8 \mathrm{~mL})$ were taken in Becton Dickinson vacutainer cell preparation tubes (Becton Dickinson, Franklin Lakes, NJ, USA) and processed in the laboratory of the Division of Immunology and Allergy (Geneva University Hospital) within $3 \mathrm{~h}$ of sampling. T-SPOT.TB analysis was performed according to the supplier's instructions. Peripheral blood mononuclear cells were separated by centrifugation, counted, resuspended in serum-free culture medium (AIM$\mathrm{V}^{\mathrm{TM}}$; Gibco, Invitrogen, Basle, Switzerland) and dispensed into wells pre-coated with an anti-IFN- $\gamma$ antibody $\left(2.5 \times 10^{5}\right.$ cells. well ${ }^{-1}$ ). Plates were incubated overnight at $37^{\circ} \mathrm{C}$ in an atmosphere of $5 \%$ carbon dioxide in the presence of medium alone (negative control), phytohaemagglutinin (positive control) or the M. tuberculosis-specific antigens ESAT-6 (panel A) and CFP10 (panel B). After incubation, the wells were washed and developed using a conjugate directed against the antibody used and an enzyme substrate. Results were analysed using an automated ELISPOT reader (AID, Strasbourg, Germany). According to the manufacturer's recommendations, tests were considered indeterminate if the results of the positive control were $<20 \mathrm{sfu}$. Results were scored as positive if the results in either the ESAT- 6 or CFP-10 wells were $>6$ sfu greater than those of the negative control. Results are reported with negative control results subtracted (i.e. measured sfu number minus sfu number of negative control), according to the recommendations of the manufacturer. The results for ESAT-6 (panel A) and CFP-10 (panel B) were also scored individually using the same procedure.

In order to determine whether T-SPOT.TB results could discriminate subjects with active TB from those with LTBI, quantitative T-SPOT.TB results in patients with TB were compared with those from subjects with LTBI. Since the aim of the present study was to establish whether a threshold sfu number could discriminate between LTBI and TB in subjects with a positive T-SPOT.TB result, LTBI was arbitrarily defined as the presence of a positive T-SPOT.TB result in contacts without any clinical symptoms or signs suggestive of TB and normal chest radiography results, irrespective of TST results. All subjects with LTBI were followed for $1 \mathrm{yr}$.

\section{Statistical analysis}

Data are presented as mean \pm SD unless otherwise specified. T-SPOT.TB results for patients with TB and LTBI are presented as boxplots (with the box showing the median and interquartile range, and the vertical bars the 5th and 95th percentiles). For subjects with LTBI, T-SPOT.TB results are presented according to TST status. Results were compared between groups using unpaired t-tests. The level of significance was set at $\mathrm{p}<0.05$.

Receiver operating characteristic (ROC) analysis was performed in order to determine a threshold sfu number for distinguishing TB from LTBI. Area under the curve (AUC) and optimal combination of Se and Sp (highest sum of Se plus Sp) are reported, as well as the $95 \%$ confidence interval (CI) of the 
AUC. ROC analysis was also performed for panel A and B (ESAT-6 and CFP-10 antigens, respectively) sfu numbers. Based on ROC curve results (threshold sfu number with optimal Se and Sp), the relationship between pre-test and posttest probability of TB was computed according to Bayes's theorem (see Appendix for details of negative and positive test results). Low, intermediate and high pre-test probabilities were defined, respectively, as $<25,25-75$ and $>75 \%$ [15].

For defining subjects with probable LTBI in contact-tracing procedures, NICE guidelines recommend a 15-mm cut-off for TST induration diameter in BCG-vaccinated individuals, and a 5-mm cut-off in nonvaccinated subjects. The Swiss guidelines acknowledge the CDC guidelines (5 mm cut-off), but recommend a 10-mm cut-off for TST induration diameter, irrespective of BCG status. ROC curve analysis was performed to simulate the two-step procedure, according to both the NICE and Swiss guidelines.

\section{RESULTS}

During the study period, 58 subjects with TB and 310 subjects screened after contact with a TB case fulfilled the inclusion criteria and agreed to be included in the study protocol. The contacts (161 male and 149 female; age (range) 40 \pm 13 (1683 ) yrs) were mostly (74.2\%) foreign-born, had been exposed to an index case with either smear-positive/culture-positive pulmonary TB $(n=270(87 \%))$, smear-negative/culture-positive pulmonary TB $(n=22(7 \%))$, smear-negative/culture-negative pulmonary TB $(n=3(1 \%))$ or extrapulmonary TB $(n=15(5 \%))$. A history of prior TST was either unreliable or unavailable in most cases; 255 (82.3\%) subjects had either a history of BCG vaccination or a suggestive scar. The TST induration diameter was $>5 \mathrm{~mm}$ in $185(60 \%)$ subjects and $>10 \mathrm{~mm}$ in $157(51 \%)$ subjects; $127(41 \%)$ had a positive T-SPOT.TB result. Seven tests were undetermined. Of all subjects with a positive T-SPOT.TB result, $84(66 \%)$ had a TST induration diameter of $>10 \mathrm{~mm}$ and $99(78 \%)$, of $>5 \mathrm{~mm}$. After $1 \mathrm{yr}$ of follow-up, none of the contacts had developed TB.

Subjects with TB (30 male and 28 female; age (range) $37 \pm 17$ (17-92) yrs) were mostly $(n=50(86 \%))$ foreign-born, were all HIV negative, and exhibited either smear-positive/culturepositive pulmonary TB $(n=37(64 \%))$, smear-negative/culturepositive pulmonary TB $(n=10(17 \%))$ or culture-positive extrapulmonary TB $(n=11 \quad(19 \%))$. The T-SPOT.TB gave positive results in $57(98 \%)$ cases, ESAT-6 in $52(89.6 \%)$ and CFP-10 in 54 (93\%).

Mean (range) sfu numbers were: $107.4 \pm 56.0$ (1-207) for TB; $53.6 \pm 60.3$ (7-239; $\mathrm{p}<0.0001$ versus TB) for subjects with LTBI and a TST induration diameter of $>5 \mathrm{~mm}$; and $18.9 \pm 27.1$ (7143; $\mathrm{p}<0.0001$ versus TB) for subjects with LTBI and a TST induration diameter of $\leqslant 5 \mathrm{~mm}$ (fig. 1).

By ROC curve analysis, the optimal sfu number for distinguishing TB from LTBI (highest sum of Se plus Sp) was 49.5, with an Se of $82.8 \%$ and an Sp of $74.2 \%$. The AUC of the ROC curve was 0.805 (SEM $0.034 ; 95 \%$ CI $0.738-0.872$; fig. 2). The AUCs of the ROC curves for panels A (ESAT-6) and B (CFP-10) were lower (table 1). Table 2 gives the Se and Sp of T-SPOT.TB at different threshold levels.

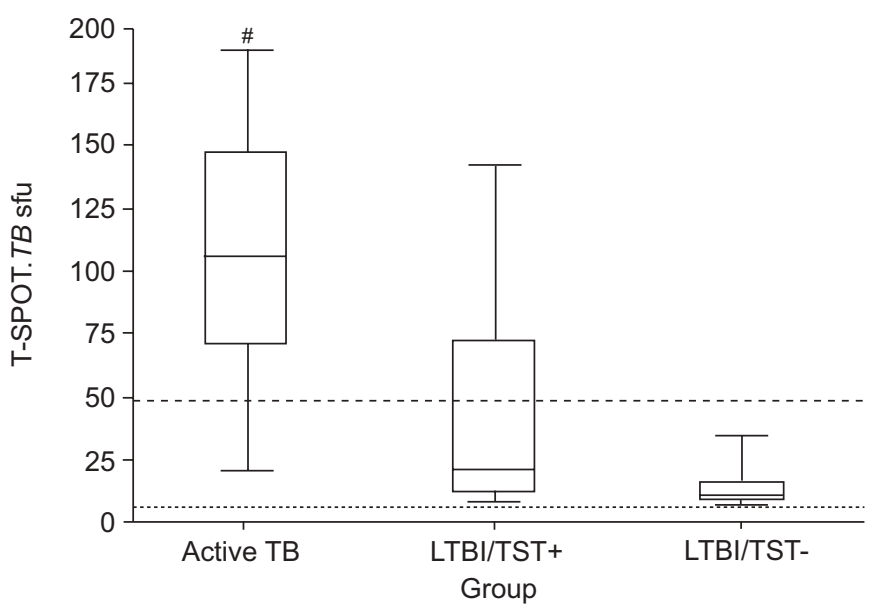

FIGURE 1. Boxplot showing spot-forming unit (sfu) numbers of patients with positive T-SPOT.TB results. Boxes represent median and interquartile range; vertical bars represent the 5th and 95th percentiles. -------: threshold determined by receiver operating characteristic analysis; $\cdots$ : manufacturer's threshold. TB: tuberculosis $(n=58)$; LTBI: latent TB infection; TST+: positive tuberculin skin-test (TST) result (induration diameter of $>5 \mathrm{~mm} ; \mathrm{n}=99$ ); TST-: negative TST result (induration diameter of $\leqslant 5 \mathrm{~mm} ; \mathrm{n}=28$ ). ${ }^{*}: \mathrm{p}<0.0001$ versus both other groups (unpaired t-test).

A ROC analysis was performed simulating the two-step procedure recommended in the Swiss and UK national guidelines, with a positive T-SPOT.TB result confirming a positive TST result (table 3 ). The optimal threshold values for distinguishing TB from LTBI (49.5 sfu) and Se (82.8\%) were unchanged. AUC and Sp were lower in all three simulations than in the model including all contacts with a positive $\mathrm{T}$ SPOT.TB result.

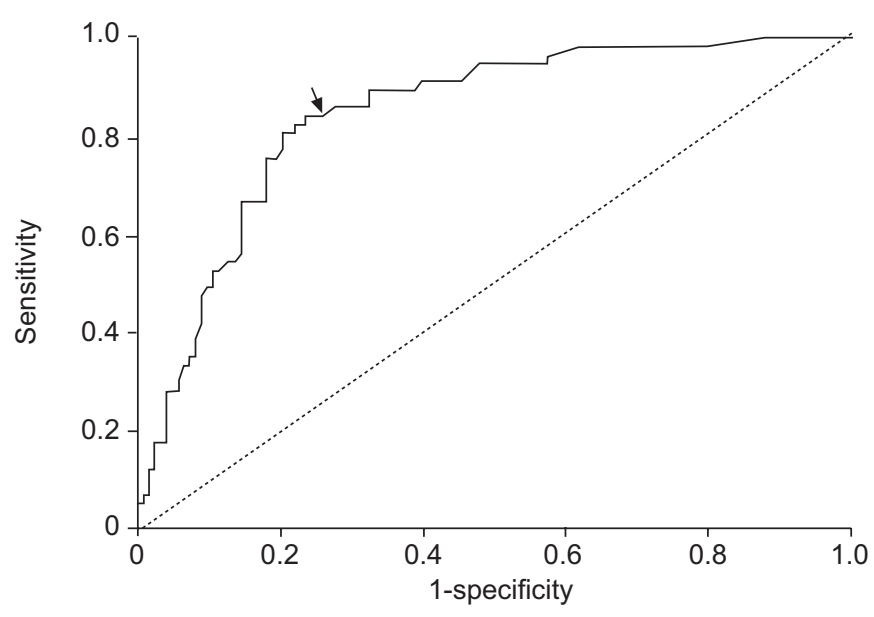

FIGURE 2. Receiver operating characteristic (ROC) curve for patients with a positive T-SPOT.TB result and either active tuberculosis (TB) or latent TB infection (LTBI). The area under the curve (AUC) is 0.805 (95\% confidence interval 0.738 0.872). The arrow indicates the point of optimal sensitivity (82.8\%) and Sp (74.2\%) for distinguishing TB from $\mathrm{LTBI}$, and corresponds to a threshold value of 49.5 spotforming units (note that this value differs from that provided by the test manufacturer). $\ldots . .$. ROC curve with an AUC of 0.5 , i.e. without any discriminatory value. 


\begin{tabular}{|c|c|c|c|c|c|}
\hline \multirow[t]{2}{*}{ TABLE 1} & \multicolumn{5}{|c|}{$\begin{array}{l}\text { Receiver operating characteristic curve analysis } \\
\text { of tuberculosis (TB) cases and contacts with } \\
\text { positive T-SPOT.TB results \# }\end{array}$} \\
\hline & AUC & $95 \% \mathrm{Cl}$ & $\begin{array}{c}\text { Threshold } \\
\text { sfu }\end{array}$ & $\begin{array}{c}\text { Sensitivity } \\
\%\end{array}$ & $\begin{array}{c}\text { Specificity } \\
\%\end{array}$ \\
\hline T-SPOT.TB & 0.805 & $0.738-0.872$ & 49.5 & 82.8 & 74.2 \\
\hline ESAT- 6 & 0.786 & $0.715-0.856$ & 8.5 & 89.7 & 58.6 \\
\hline CFP-10 & 0.771 & $0.692-0.849$ & 58.0 & 70.7 & 80.5 \\
\hline \multicolumn{6}{|c|}{$\begin{array}{l}\text { T-SPOT.TB analysis was performed using the antigens } 6-\mathrm{kDa} \text { early secretory } \\
\text { antigenic target (ESAT-6) and culture filtrate protein (CFP) } 10 \text {, both together and } \\
\text { separately. AUC: area under the curve; Cl: confidence interval; sfu: spot- } \\
\text { forming unit. } \# \text { : } 58 \text { TB cases and } 127 \text { contacts; } \because \text { : sfu number giving the optimal } \\
\text { combination (highest sum) of sensitivity and specificity. }\end{array}$} \\
\hline
\end{tabular}

\begin{tabular}{|c|c|c|c|c|}
\hline TABLE 2 & $\begin{array}{l}\text { Receiver } \\
\text { of sensit } \\
\text { T-SPOT. }\end{array}$ & $\begin{array}{l}\text { perating c } \\
\text { ty and spe } \\
3 \text { results }\end{array}$ & $\begin{array}{l}\text { racteristic } \\
\text { icity at diffe }\end{array}$ & $\begin{array}{l}\text { e analysis } \\
\text { it threshold }\end{array}$ \\
\hline \multirow{2}{*}{$\begin{array}{l}\text { Threshold } \\
\text { sfu }\end{array}$} & \multirow{2}{*}{$\begin{array}{c}\text { Sensitivity } \\
\%\end{array}$} & \multirow{2}{*}{$\begin{array}{c}\text { Specificity } \\
\%\end{array}$} & \multicolumn{2}{|c|}{ Likelihood ratio } \\
\hline & & & $\begin{array}{l}\text { Positive } \\
\text { T-SPOT.TB }\end{array}$ & $\begin{array}{l}\text { Negative } \\
\text { T-SРОт.TB }\end{array}$ \\
\hline 13.5 & 94.8 & 41.4 & 1.6 & 0.1 \\
\hline $49.5^{+}$ & 82.8 & 74.2 & 3.2 & 0.2 \\
\hline 136.5 & 32.8 & 90.6 & 3.5 & 0.7 \\
\hline 180.5 & 12.1 & 95.3 & 2.6 & 0.9 \\
\hline
\end{tabular}

sfu: spot-forming unit. ${ }^{\#}$ : see table $1 ; "$ : analysis included 58 TB cases and 127 contacts with positive T-SPOT.TB results; ${ }^{+}$: optimal threshold.

Figure 3 and table 4 show the relationship between the preand post-test probability of active TB, according to Bayes's theorem (see Appendix), with either a high or low T-SPOT.TB sfu number (i.e. above or below threshold of $49.5 \mathrm{sfu}$ ). The likelihood ratio of TB was 3.2 with a T.SPOT.TB result of $>49.5 \mathrm{sfu}$ versus 0.2 for a result of $<49.5 \mathrm{sfu}$. As shown in figure 3 , an intermediate pre-test probability of TB $(25-75 \%)$ is associated with a $7-40 \%$ post-test probability of TB if sfu number is $<49.5$, and a $50-90 \%$ post-test probability of TB if it is $>49.5$ (fig. 3).

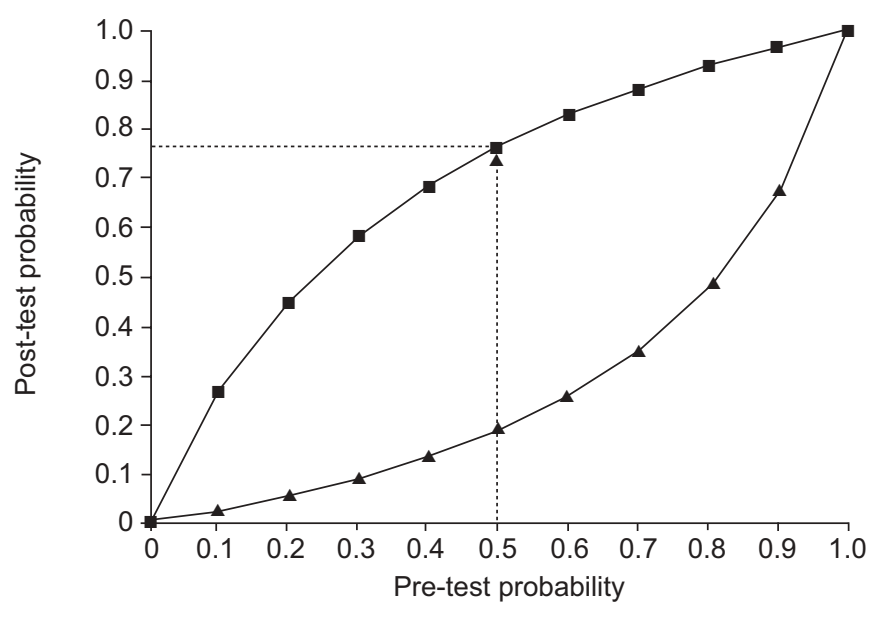

FIGURE 3. Relationship between pre- and post-test probabilities of active tuberculosis (TB; Bayesian analysis) with a T-SPOT.TB result either above ( likelihood ratio 3.2) or below ( $\boldsymbol{\Lambda}$ : likelihood ratio 0.2) the threshold of 49 spot-forming units (sfu) defined by receiver operating characteristic curve analysis. The pre-test probability of TB was determined from the medical history and examination. The posttest probability was determined according to whether the T-SPOT.TB result was above or below $49.5 \mathrm{sfu}$. -------: hypothetical situation with a pre-test probability of 0.5 and a T-SPOT.TB result of $>49.5$ sfu (post-test probability of 0.76 )

\section{DISCUSSION}

The aim of the present study was to determine whether quantitative analysis using the T-SPOT.TB IFN- $\gamma$ assay (with TSPOT.TB results as a continuous variable) could discriminate between active and latent TB infection in immunocompetent adults with a positive T-SPOT.TB result. The present results show that there is an apparent relationship between activity of $M$. tuberculosis infection and secretion of IFN- $\gamma$ by lymphocytes, as assessed by the T-SPOT.TB, with significantly higher IFN- $\gamma$ levels being associated with active disease. However, there was considerable overlap of the quantitative results of the T-SPOT.TB between subjects with TB and those with probable LTBI (fig. 1). ROC curve analysis shows that, in individuals with positive test results, the T-SPOT.TB has an Se of $82.8 \%$ and an $\mathrm{Sp}$ of $74.2 \%$ for distinguishing active from latent disease at a threshold of 49 sfu (table 1; fig. 2). Using Bayesian analysis, in a clinical situation of intermediate pretest probability (i.e. a contact with normal clinical examination and chest radiographic results, but with a history of recent weight loss and nocturnal sweating), a T-SPOT.TB result of $<49 \mathrm{sfu}$ is associated with a post-test probability of TB of

TABLE 3 Receiver operating characteristic (ROC) curve analysis simulating the two-step procedure in contact-tracing

\begin{tabular}{lccccc} 
& AUC & $\mathbf{9 5 \%} \mathbf{C l}$ & Threshold ${ }^{*}$ sfu & Sensitivity \% & Specificity \% \\
\hline TST $>\mathbf{5}$ mm/T-SPOT.TB+ & 0.787 & $0.715-0859$ & 49.5 & 82.8 & 70 \\
TST $>$ 10 $\mathbf{m m} /$ T-SPOT.TB+ & 0.676 & $0.574-0.777$ & 49.5 & 82.8 & 68.1 \\
NICE guidelines & 0.771 & $0.695-0.847$ & 49.5 & 82.8 & 54.4 \\
\hline
\end{tabular}

The analysis was performed according to Swiss guidelines, at cut-off tuberculin skin test (TST) induration diameters of 5 ( $n=157)$ and $10 \mathrm{~mm}(\mathrm{n}=142)$, and according to National Institute for Health and Clinical Excellence (NICE) guidelines (see Statistical analysis section; $n=115$ ). AUC: area under the curve; Cl: confidence interval; sfu: spot-forming unit. \#: sfu number giving the optimal combination (highest sum) of sensitivity and specificity for distinguishing active from latent tuberculosis infection. 


\begin{tabular}{|c|c|c|c|}
\hline TABLE 4 & \multicolumn{3}{|c|}{$\begin{array}{l}\text { Bayesian analysis: illustration of post-test } \\
\text { probability of tuberculosis (TB) according to pre- } \\
\text { test probability and quantitative T-SPOT.TB result }\end{array}$} \\
\hline \multirow{2}{*}{\multicolumn{2}{|c|}{$\begin{array}{l}\text { Pre-test } \\
\text { probability }\end{array}$}} & \multicolumn{2}{|c|}{ Post-test probability \% } \\
\hline & & T-SPOT.TB $<49.5 \mathrm{sfu}$ & T-SPOT.TB $>49.5 \mathrm{sfu}$ \\
\hline Low (20\%) & & 5 & 45 \\
\hline Intermediate & (50\%) & 19 & 76 \\
\hline High (80\%) & & 48 & 93 \\
\hline \multicolumn{4}{|c|}{$\begin{array}{l}\text { All subjects had a positive T-SPOT.TB result according to the manufacturer's } \\
\text { criteria (see Patients and methods section). The threshold value of } 49.5 \text { spot- } \\
\text { forming units (sfu) was determined by receiver operating characteristic curve } \\
\text { analysis (tables } 1 \text { and 2; fig. 2). The pre-test probability was estimated from the } \\
\text { medical history and symptoms (i.e. weight loss, nocturnal sweating, importance } \\
\text { of exposure to index case). }\end{array}$} \\
\hline
\end{tabular}

$7-40 \%$, whereas a result above this threshold yields a post-test probability of TB of 50-90\% (fig. 3). Thus, although higher sfu numbers increase the probability of active disease (table 2), the T-SPOT.TB cannot be used in clinical practice to formally establish or exclude a diagnosis of active TB.

In the present study, the focus was on the contribution of T-SPOT.TB to distinction between active and latent TB in subjects with probable LTBI. An arbitrary definition was used to define LTBI (positive T-SPOT.TB result without clinical or radiological evidence of $\mathrm{TB}$ ); this definition can be challenged, since, to date, no large longitudinal studies have been performed quantifying the lifetime risk of developing active TB in subjects with a positive IGRA result. Thus, a ROC analysis was performed simulating the two-step procedure recommended in the Swiss and UK NICE guidelines. The optimal threshold sfu number for distinguishing TB from LTBI and Se was unchanged, whereas Sp and the AUC decreased while simulating either the NICE guidelines or a two-step procedure with a TST cut-off induration diameter of $10 \mathrm{~mm}$ (table 3). Response to either antigen (ESAT-6 or CFP-10) individually did not improve the discriminatory performance of the assay (table 1). Other reports have explored the use of IGRAs in the diagnosis of TB. LEE et al. [16] studied 87 subjects with active TB (culture proven in 63\%) and 131 young students with a low risk of LTBI. The AUCs of the ROC curves were not reported; Se $(92 \%$; 95\% CI 84-97\%) and Sp (91.6\%; 95\% CI 85$96 \%$ ) were higher than reported in the present study. However, the relevance of these results is debatable since, for the clinician, the challenge is not to distinguish between subjects who are naive to $\mathrm{TB}$ exposure versus active $\mathrm{TB}$, but rather to distinguish active from latent disease among subjects who probably have been infected. Several studies have consistently shown higher production of IFN- $\gamma$ by lymphocytes challenged by $M$. tuberculosis-specific antigens in culture-proven active TB than in LTBI [16-19]. GOLETTI et al. [19] compared the results of different IGRAs in patients with clinical suspicion of TB according to outcome (culture-proven $\mathrm{TB}$ versus alternative diagnosis established or resolution of clinical symptoms without drugs active against TB). As in the present study, sfu numbers for selected peptides or intact ESAT-6 and CFP-10 were significantly higher in patients with TB than in TSTpositive or -negative controls. The quantitative results of either

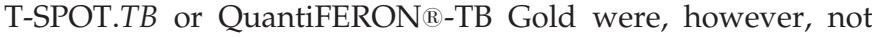
reported.

Several limitations of the present study should be mentioned. First, the population selected for the present study should ideally have included TB cases found among persons screened. This, however, was not possible for obvious epidemiological reasons; in developed countries, a $1-2 \%$ rate of TB would be expected in this population, thus rendering such a design impossible [10]. Indeed, in Geneva, only $0.2 \%$ of subjects screened show active TB; these subjects contribute to $<1 \%$ of total cases of TB in the Geneva area. It was surmised that IFN- $\gamma$ production by peripheral lymphocytes would be similar in TB cases, whether or not cases were detected among contacts. However, it may be hypothesised that TB cases detected in a contact-tracing setting could be at an earlier stage of their infection, and thus exhibit lower sfu numbers.

Secondly, the present definition of LTBI was arbitrary. Indeed, there is no gold standard for defining LTBI. IGRA results correlate better with exposure than do TST results, but the lifetime risk of active TB based on IGRA results alone is unknown [4-6]. It was decided to study the clinical situation resulting from the present CDC, NICE and Swiss recommendations; subjects considered as having probable LTBI were detected by a positive IGRA result with or without a previous TST. The question is, therefore, in this group of subjects, can IGRAs detect active TB? Studying positive T-SPOT.TB subjects alone or TSPOT.TB- and TST-positive subjects did not significantly alter the present results according to prevailing guidelines.

Thirdly, because there is no gold standard for the diagnosis of LTBI, TB cases may have been missed among subjects with presumed LTBI. However, this is very unlikely, since all of the subjects underwent a normal clinical examination and chest radiography. Furthermore, the expected rate of TB among the 127 contacts in the Geneva area with LTBI would be one case at most. Indeed, no missed case of TB was detected over $1 \mathrm{yr}$ of follow-up.

Finally, the present study was not designed to address the issue of using IGRAs for excluding TB, since all of the subjects studied gave a positive IGRA result (it is of note that the Se of the T-SPOT.TB in the present study was $98 \%$ for TB).

One unexplained observation is the difference in T-SPOT.TB results observed between subjects with a TST induration diameter of $>5 \mathrm{~mm}$ versus those with an induration diameter of $\leqslant 5 \mathrm{~mm}$ (fig. 1). A TST induration diameter of $\leqslant 5 \mathrm{~mm}$ in immunocompetent adults exposed to TB would suggest absence of LTBI, and therefore false-positive results of the TSPOT.TB assay. Indeed, the available epidemiological data suggest that these subjects are at low risk of developing active TB [20]. However, no longitudinal data are available yet to assess the risk of developing active TB in TST-negative T-SPOT.TB-positive individuals.

In summary, the present study confirms previous observations that higher production of interferon- $\gamma$, measured by interferon$\gamma$ release assay, correlates to some extent with the activity of 
Mycobacterium tuberculosis infection. However, the sensitivity and specificity of the T-SPOT.TB assay for distinguishing active versus latent tuberculosis are too low to recommend the use of any particular threshold value for confirming or excluding active disease. These results may justify a higher suspicion index of active tuberculosis in patients with very high numbers of spot-forming units (table 2). Longitudinal studies of subjects not treated for latent tuberculosis infection would be necessary in order to confirm a higher risk of active tuberculosis in patients with high numbers of spot-forming units.

\section{APPENDIX: BAYES'S THEOREM}

Bayesian analysis was used to compute figure 3. If T-SPOT.TB results were positive $(>49.5 \mathrm{sfu})$, the post-test probability of active TB (Ppost) was calculated as follows:

$$
P_{\text {post }}=\text { PreSe } /((\text { preSe })+(1-\text { Pre })(1-S p))
$$

where Ppre is the pre-test probability of active TB. If T-SPOT.TB results were negative $(<49.5 \mathrm{sfu})$, the Ppost was calculated as follows:

$$
P_{\text {post }}=\text { Ppre }(1-\text { Se }) / \text { pre }(1-\text { Se })+(1-\text { Pre }) \text { Sp }
$$

\section{REFERENCES}

1 Diel R, Ernst M, Doscher G, et al. Avoiding the effect of BCG vaccination in detecting Mycobacterium tuberculosis infection with a blood test. Eur Respir J 2006; 28: 16-23.

2 Pai M, Menzies D. Interferon-gamma release assays: what is their role in the diagnosis of active tuberculosis? Clin Infect Dis 2007; 44: 74-77.

3 Richeldi L. An update on the diagnosis of tuberculosis infection. Am J Respir Crit Care Med 2006; 174: 736-742.

4 Shams H, Weis SE, Klucar P, et al. Enzyme-linked immunospot and tuberculin skin testing to detect latent tuberculosis infection. Am J Respir Crit Care Med 2005; 172: 1161-1168.

5 Zellweger JP, Zellweger A, Ansermet S, de Senarclens B, Wrighton-Smith P. Contact tracing using a new T-cellbased test: better correlation with tuberculosis exposure than the tuberculin skin test. Int J Tuberc Lung Dis 2005; 9: 1242-1247.

6 Lalvani A, Pathan AA, Durkan H, et al. Enhanced contact tracing and spatial tracking of Mycobacterium tuberculosis infection by enumeration of antigen-specific T cells. Lancet 2001; 357: 2017-2021.

7 Mazurek GH, Jereb J, LoBue P, Iademarco MF, Metchock B, Vernon A. Guidelines for Using the QuantiFERON®-TB Gold Test for Detecting Mycobacterium tuberculosis Infection, United States. MMWR Morb Mortal Wkly Rep 2005; 54: 49-62.

8 National Institute for Health and Clinical Excellence. Tuberculosis. Clinical Diagnosis and Management of
Tuberculosis, and Measures for its Prevention and Control. www.nice.org.uk/CG033 Date last updated: March 2006. Date last accessed: July 31, 2007.

9 Brändli O, Desgrandchamps D, Gabathuler U, et al. Manuel de la Tuberculose. [Manual of Tuberculosis.] http:/ / tbinfo. $\mathrm{ch} / \mathrm{fr} /$ presentations/manuel-de-la-tuberculose.html Date updated: March 2007. Date last accessed: August 2, 2007.

10 Anon. American Thoracic Society/Centers for Disease Control and Prevention/Infectious Diseases Society of America: controlling tuberculosis in the United States. Am J Respir Crit Care Med 2005; 172: 1169-1227.

11 Aiken AM, Hill PC, Fox A, et al. Reversion of the ELISPOT test after treatment in Gambian tuberculosis cases. BMC Infect Dis 2006; 6: 66.

12 Carrara S, Vincenti D, Petrosillo N, Amicosante M, Girardi E, Goletti D. Use of a T cell-based assay for monitoring efficacy of antituberculosis therapy. Clin Infect Dis 2004; 38: 754-756.

13 Pathan AA, Wilkinson KA, Klenerman P, et al. Direct ex vivo analysis of antigen-specific IFN- $\gamma$-secreting CD4 T cells in Mycobacterium tuberculosis-infected individuals: associations with clinical disease state and effect of treatment. J Immunol 2001; 167: 5217-5225.

14 Nicol MP, Pienaar D, Wood K, et al. Enzyme-linked immunospot assay responses to early secretory antigenic target 6 , culture filtrate protein 10, and purified protein derivative among children with tuberculosis: implications for diagnosis and monitoring of therapy. Clin Infect Dis 2005; 40: 1301-1308.

15 Mayer D. Bayes' theorem, predictive values, post-test probabilities, and interval likelihood ratios. In: Mayer D, ed. Essential Evidence-Based Medicine. Cambridge, Cambridge University Press, 2004; pp. 222-236.

16 Lee JY, Choi HJ, Park IN, et al. Comparison of two commercial interferon- $\gamma$ assays for diagnosing Mycobacterium tuberculosis infection. Eur Respir J 2006; 28: 24-30.

17 Ravn P, Munk ME, Andersen AB, et al. Prospective evaluation of a whole-blood test using Mycobacterium tuberculosis-specific antigens ESAT-6 and CFP-10 for diagnosis of active tuberculosis. Clin Diagn Lab Immunol 2005; 12: 491-496.

18 Goletti D, Carrara S, Vincenti D, et al. Accuracy of an immune diagnostic assay based on RD1 selected epitopes for active tuberculosis in a clinical setting: a pilot study. Clin Microbiol Infect 2006; 12: 544-550.

19 Goletti D, Vincenti D, Carrara S, et al. Selected RD1 peptides for active tuberculosis diagnosis: comparison of a gamma interferon whole-blood enzyme-linked immunosorbent assay and an enzyme-linked immunospot assay. Clin Diagn Lab Immunol 2005; 12: 1311-1316.

20 Rieder H. Epidemiological Basis of Tuberculosis Control. 1st Edn. Paris, International Union Against Tuberculosis and Lung Disease, 1999. 